\title{
Accurate optical positions for 2978 objects from the Second Byurakan Survey (SBS) with the Digitized Sky Survey*
}

\author{
M.D. Bicay ${ }^{1}$, J.A. Stepanian ${ }^{2}$, V.H. Chavushyan ${ }^{3}$, L.K. Erastova ${ }^{4}$, V.T. Ayvazyan ${ }^{5}$, J. Seal ${ }^{6}$, and G. Kojoian ${ }^{7, \star \star ~}$ \\ 1 Jet Propulsion Laboratory, 4800 Oak Grove Drive, MS 126-304, Pasadena, CA 91109-8099, U.S.A. \\ 2 Special Astrophysical Observatory RAS, Nizhnij Arkhyz, Karachai-Cherkessia 357147, Russia \\ 3 Instituto Nacional de Astrofísica Optica Electrónica, Apartado Postal 51 y 216, CP. 72000, Puebla, Pue., Mexico \\ 4 Byurakan Astrophysical Observatory, Armenia \\ 5 Department of Physics and Astronomy, Armenian State Pedagogical Institute, Yerevan, Khangian 5, 375010, Armenia \\ ${ }^{6}$ Cambridge CR \& D, 2205 CR 1100N, Sidney, IL 61877, U.S.A. \\ 7 Department of Physics and Astronomy, University of Wisconsin-Eau Claire, WI 54702-4004, U.S.A. \\ e-mail: mbicay@sirtfweb.jpl.nasa.gov, jstep@sao.ru,vahram@inaoep.mx, lke@bao.sci.am, ayvo@sao.ru
}

Received February 12, 1998; accepted March 13, 2000

\begin{abstract}
Optical positions of 2978 objects listed in the Second Byurakan Survey (SBS) were obtained using the Digitized Sky Survey (DSS), and are given with an rms uncertainty $\sim 1$ arcsec in each coordinate.
\end{abstract}

Key words: surveys — catalogs: active — galaxies — quasars

\section{Introduction}

In 1960 Markarian initiated an objective prism survey of the northern sky (Markarian 1967). It was the first survey in which a thin objective prism was used for selecting peculiar extragalactic objects characterized by an ultraviolet emission excess. The observations were made with the $40^{\prime \prime}-52^{\prime \prime}$ Schmidt camera of the Byurakan Observatory, equipped with 1.5, 3 and 4 degree objective prisms. This survey become known as the First Byurakan Survey (FBS) (see for complete references Markarian et al. 1981). The FBS covered most of the sky visible from the Byurakan Observatory in Armenia. Ten years later a similar survey in the southern sky was undertaken by Smith (1975) at the CTIO in Chile.

The FBS (the "Markarian survey") yielded a complete sample of AGNs down to limiting magnitude $m_{\mathrm{pg}}=$

Send offprint requests to: J.A. Stepanian

* Tables 1 and 2 are only available in electronic form at the CDS via anonymous ftp 130.79.128.5 or via

http://cdsweb.u-strasbg.fr/Abstract.html

$\star \star$ It is with great sadness that we report the death of one of the iniciators of this work, Gabriel Kojoian, in 1998.

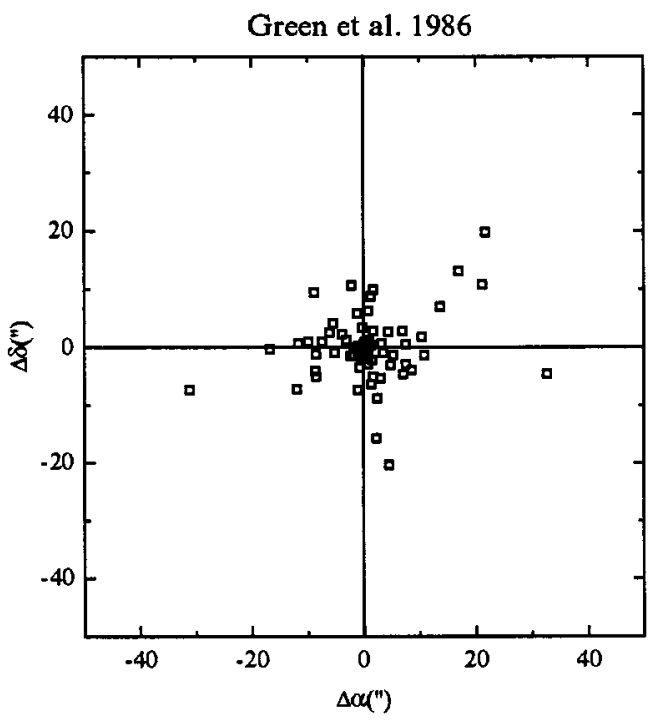

Fig. 1. A plot of the difference in RA and Declination between the newly measured positions and the previously available optical position of PG objects (Green et al. 1986)

15.5 . Subsequent optical spectroscopy revealed that over 200 Seyfert galaxies, 13 QSOs, 3 BL Lac objects and hundreds of starburst, blue compact and HII galaxies were cataloged in the FBS.

In 1974 the Second Byurakan Survey (SBS) was initiated. It was completed in 1991 (Markarian \& Stepanian 1983, 1984a,b; Markarian et al. 1985, 1986; Stepanian et al. 1988, 1990; Stepanian 1994). The same Schmidt camera was used for observations. The SBS covered a smaller region of the northern sky of about 1000 sq. degrees bounded by: $07^{\mathrm{h}} 40^{\mathrm{m}}<\alpha<17^{\mathrm{h}} 15^{\mathrm{m}}$ 
Table 3. Comparaison between our and "other" measurements

\begin{tabular}{|c|c|c|c|c|c|c|}
\hline \multirow[t]{3}{*}{ Reference } & & & \multicolumn{2}{|c|}{ Previous original measurements } & \multicolumn{2}{|c|}{ New DSS measurements } \\
\hline & \multicolumn{2}{|c|}{ Accuracy } & \multirow[t]{2}{*}{ Number } & \multirow[t]{2}{*}{ Notes } & \multicolumn{2}{|c|}{ Mean deviation } \\
\hline & $\overline{\mathrm{RA}}$ & Dec. & & & $\mathrm{RA}$ & Dec. \\
\hline Green et al. (1986) & 8.7 & $7^{\prime \prime} 6$ & 150 & POSS (KPNO Grand Mashine) & $15^{\prime \prime}$ & $15^{\prime \prime}$ \\
\hline Sanduleak \& Pesch (1987) & $3 . \prime 0$ & $6 . .0$ & 100 & POSS (Warner \& Swasey obs.) & $2^{\prime \prime} .5$ & $3 .{ }^{\prime \prime} 0$ \\
\hline Nelson et al. (1988) & $1^{\prime \prime} .5$ & $1^{\prime \prime} 5$ & 118 & $\mathrm{PDS}$ & $1^{\prime \prime} .5$ & $1^{\prime \prime} 5$ \\
\hline Seal et al. (1990) & $1^{\prime \prime} .5$ & $1^{\prime \prime} 5$ & 134 & POSS & $1^{\prime \prime} .5$ & $1^{\prime \prime} .5$ \\
\hline Simpson et al. (1990) & $2 . .0$ & 2.0 & 106 & PDS & $3 .{ }^{\prime \prime} 0$ & 3.0 \\
\hline Stepanian (1994) & $<1^{\prime \prime} .5$ & $<1^{\prime \prime} .5$ & 205 & POSS (KPNO Grand Mashine) & $<1^{\prime \prime} .5$ & $<1^{\prime \prime} .5$ \\
\hline Bowen et al. (1994) & $<1^{\prime \prime} 0$ & $<1^{\prime \prime} .0$ & 9 & DSS & $<1^{\prime \prime} 0$ & $<1^{\prime \prime} .0$ \\
\hline Véron-Cetty \& Véron (1996) & $0 . .6$ & $0^{\prime \prime} 6$ & 105 & DSS & $<1_{.}^{\prime \prime} 0$ & $<1^{\prime \prime} .0$ \\
\hline SBS original lists & 1.5 & 1.5 & 741 & POSS & & \\
\hline
\end{tabular}

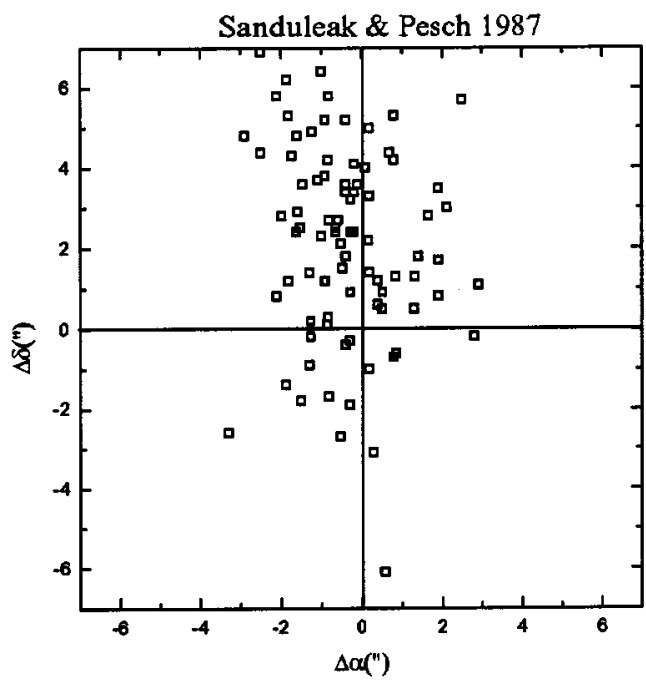

Fig. 2. Another similar plot of the differences between our position and the ones measured by Sanduleak and Pesch, indicated a standard deviation of about $2^{\prime \prime}$ in both coordinates, and systematic deviation by Dec. of about $+3^{\prime \prime}$

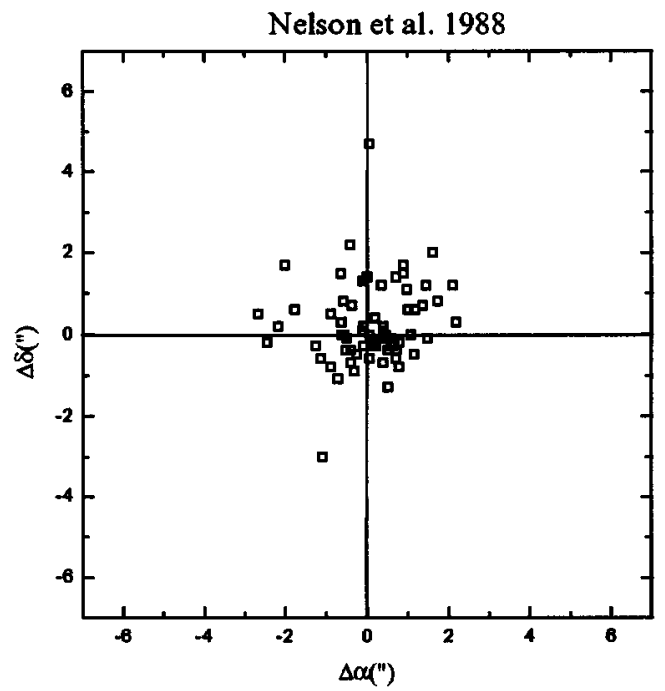

Fig. 3. A plot of the differences between our measured position, and the ones by Nelson et al. (1988), indicates a standard deviation of about $1^{\prime \prime} 5$ in both RA and Dec.

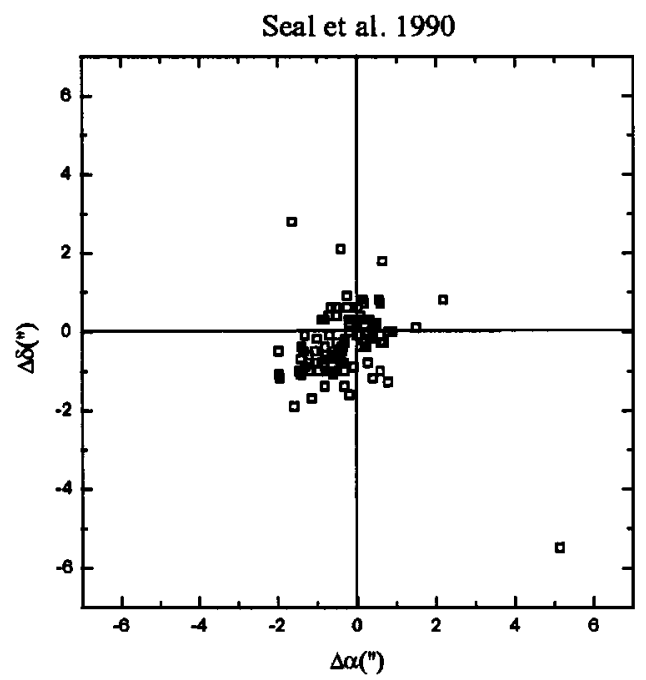

Fig. 4. A plot of the differences between our measured position and the ones measured by Seal et al. (1990), indicates a standard deviation of about $1^{\prime \prime} .5$ in both and Dec.

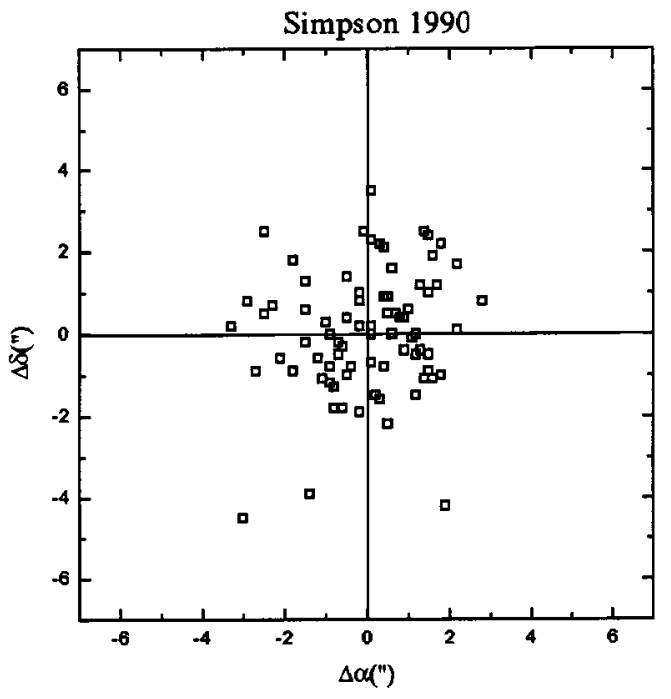

Fig. 5. A similar plot of the differences between our positions and the ones measured by Simpson (1990), indicates a standard deviation larger than $3^{\prime \prime} 0$ in both coordinates 


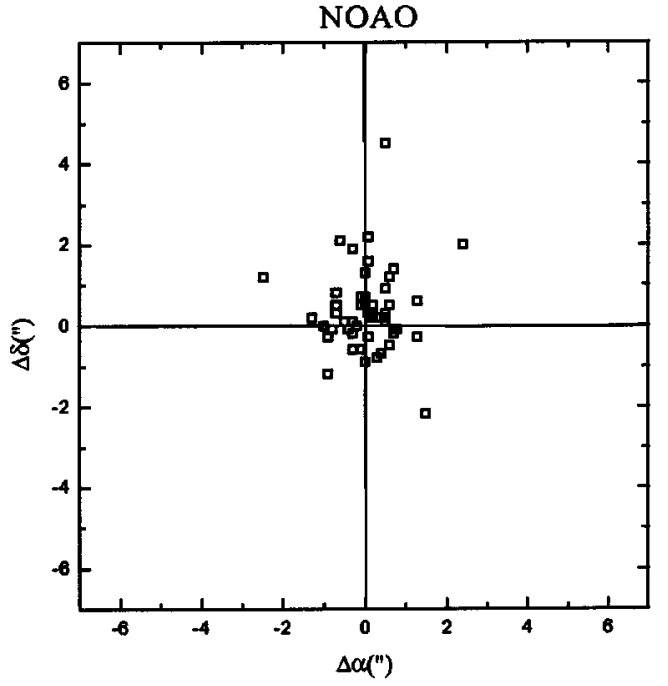

Fig. 6. A plot of the differences in RA and Dec. between the newly measured positions and the previously measured optical position of SBS objects by Stepanian et al. (1994)

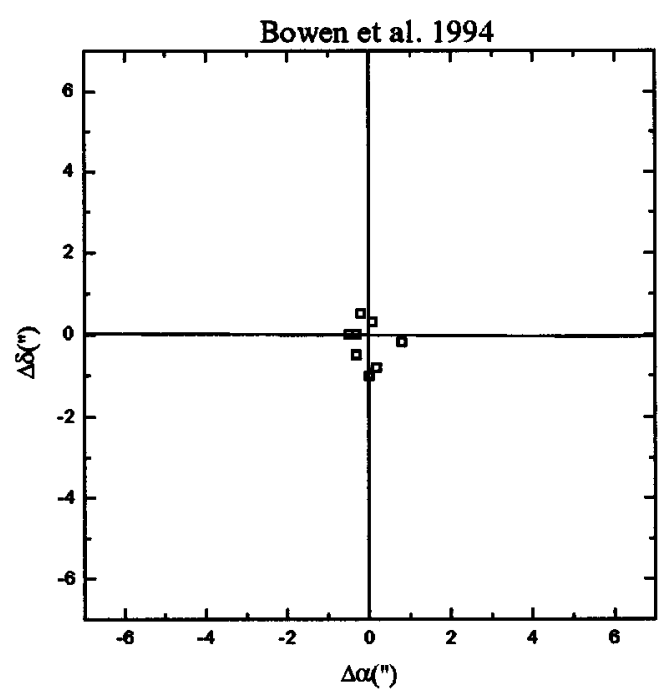

Fig. 7. A similar plot of the differences between our positions and the ones measured by Bowen et al. (1994), indicates a standard deviation smaller than $1^{\prime \prime}$ in both coordinates

and $+49^{\circ}<\delta<+61^{\circ}$. The advantage of the SBS is fainter limiting magnitude of $m_{\mathrm{pg}}=19.5$. The fainter detection limit of the SBS is partly due to an improved photographic sensitivity to the UV-excess continuum and to the relevant emission lines. This resulted in a larger surface density of the SBS objects (by about 15 times) in comparison to the FBS.

Selection of the SBS objects was done by visual inspection of plates based on the following criteria: (i) the presence of strong UV continuum, and (ii) the presence of emission lines and/or a peculiar energy distribution in the spectra. The main goal of the SBS was to indentify and characterize peculiar objects belonging to the

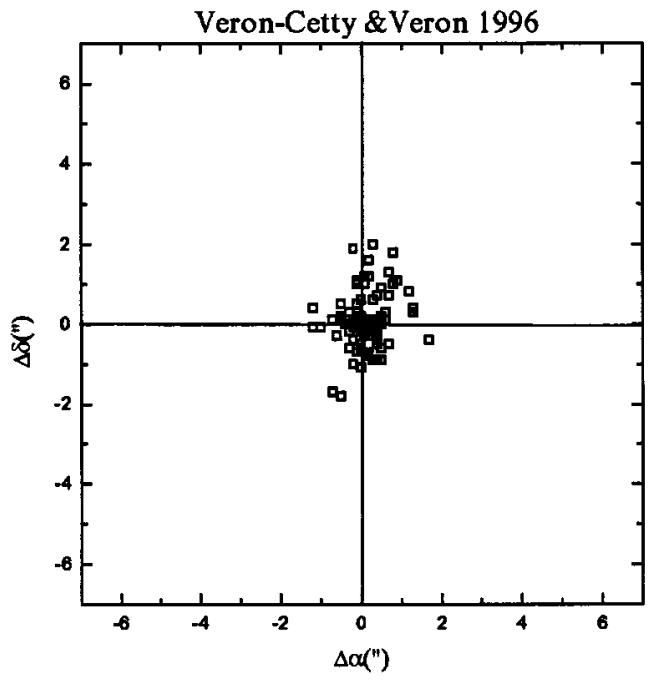

Fig. 8. A similar plot of the differences between our positions and the ones measured by Véron-Cetty \& Véron (1996), indicates a standard deviation less than $1^{\prime \prime}$ in both coordinates

following classes: (a) galaxies with UV-excess continuum radiation, (b) emission line galaxies, without appreciable UV-excess continuum, (c) QSOs and AGN candidates, (d) white dwarfs and subdwarf stars, and (e) other types of objects with strong UV continuum radiation.

Up to now $\sim 1700$ galaxies and $\sim 1800$ star-like objects with UV excess have been detected in the SBS survey. The SBS catalog will allow to derive a complete sample of faint AGNs down to $B \leq 17^{\mathrm{m}}$ 5. It should also allow the investigation of the luminosity function of QSOs, AGNs, and UV-excess galaxies, and contribute to our understanding of the large-scale structure in the Universe at scale $\leq 500 \mathrm{Mpc}$.

For any follow-up investigation of nearly 3500 SBS objects, the knowledge of an accurate optical positions is required. In this paper we present accurate optical coordinates for 2978 objects listed in the SBS catalog.

\section{Measurement procedure and the accuracy of measurements}

All measurements were made using the Digitized Palomar observatory Sky Survey (DSS) which is constructed from 6.5 degree square plates which have been digitized with a modified PDS microdensitometer. The resultant pixel size is nominally 25 microns, corresponding to about $1^{\prime \prime} .7$ arcsec.

While the primary motivation for the STScI/DSS was to support Hubble Space Telescope operations, the entire astronomical community has benefited from this service. Earlier the DSS has already been used to derive accurate coordinates for several hundred QSOs (Schneider et al. 1992; Kirhakos et al. 1994), and AGNs (Véron-Cetty \& Véron 1996). 
Two groups of researchers made an independent measurement of positions of 2978 SBS objects. One group used the facilities of the Special Astrophysical Observatory (SAO), Russia. The second group used the resources available at the Space Telescope Science Institute (STScI) in Baltimore, Maryland, U.S.A.

The measurements were made prior to the nearuniversal accessibility of the DSS over the Internet (e.g., http://archive.stsci.edu/dss/). Investigators at the SAO used the FITSview (Flexible Image Transport System) softwave (NRAO; Bill Cotton, http://www.cv.nrao.edu/bcotton/fitsview.html). It allows the user to select and display images, to mark the position of objects and to measure the coordinates and pixel brightness of the image. FITSview software was used to display the extracted images from the SAO set of DSS CDroms and, after marking of the positions of identified objects, to measure the accurate coordinate of objects for J2000.0 epoch. The coordinates were converted to B1950.0 epoch by the use of the conversion program described in Lieske (1979). Finding charts of the SBS objects were used to confirm selected objects to be measured.

Independent measurements at the STScI were made by using the DSS, after performing additional extractions of smaller spatial extent, with data resampled to a pixel size of $\sim 0^{\prime \prime} .4$ arcsec. In order to facilitate convenient electronic access to the STScI/DSS, all image data were compressed using a lossy technique. The data were typically compressed by a factor of 10 , a level that does not affect the fidelity of astrometry measurements. In all cases the astrometry relied on stars listed in the AGK3 (Heckman et al. 1975). The difference of positions between the two sets of measurement has an rms of $\sim 1^{\prime \prime} .5$ arcsec in both coordinates implying that the accuracy of each set of measurement is $\sim 1$.' 1 arcsec.

The accurate optical coordinates of the SBS objects (mean of the two sets of measurements) are listed in Tables 1 and 2. Column 1 gives the SBS designation of the objects. Columns 2 and 3 list the B1950.0 right ascension and declination, while the corresponding J2000.0 coordinates are listed in Cols. 4 and 5. An optical magnitude is listed in Col. 6. If the precision is specified in multiples of $0.01 \mathrm{mag}$, the listed value is a photometric measurement with an accuracy $<0$. 05 (Chavushyan et al. 1995); otherwise the value represents an eye estimation of the photographic magnitude with an uncertainty of 0.5 (Stepanian 1994). Finally, alternative names for the SBS objects are listed in Col. 7.

Earlier, in various astrometric programs, accurate optical positions were measured for more than 1000 out of 2978 SBS objects (Green et al. 1986; Sanduleak \& Pesch 1987; Nelson et al. 1988; Seal et al. 1990; Simpson 1990; Stepanian 1994; Bowen et al. 1994; Véron-Cetty \& Véron 1996).
The comparison of the position measured by us with the mentioned ones is presented in Table 3. Plots of the differences of positions are shown in Figs. 1-8.

\section{Results}

Optical coordinates with an accuracy of $\sim 1$ arcsec have been measured for 2978 SBS objects.

Rms deviation between the coordinates listed in original SBS papers and the newly measured positions is 1.5 arcmin in both RA and Dec.

The coordinates listed for PG objects which were determined from blue plates, then converted to celestial equatorial coordinates through the method as described in Green \& Morril (1978), show an rms deviation with respect to the newly measured positions of about $15^{\prime \prime}$ both in RA and Dec.

Case - coordinates were derived from measurements made on the IIIaJ objective-prism plates using the $x, y$ measuring machine of Warner and Swasey Observatory. The rms difference is \pm 2.5 in RA and $3^{\prime \prime} .0$ in Dec., but there is a systematic deviation of about $+3^{\prime \prime}$ by Dec.

The comparison of our positions with the most accurate position measurements (Bowen et al. 1994; VéronCetty \& Véron 1996) (see Table 3) shows that the accuracy of our measurements is about $1^{\prime \prime}$.

Acknowledgements. The STScI/DSS was produced at the Space Telescope Science Institute under US government grant NAGW-2166. The survey images are based on photographic data obtained using the Oschin Schmidt Telescope on Palomar Mountain, and were processed into compressed digital form with the permission of Palomar Observatory. The National Geographic Society - Palomar Observatory Sky Survey (POSS-I) was made by the California Institute of Technology with grants from the National Geographic Society.

The US-based investigators wish to acknowledge the assistance of Daniel Golombek (STScI).

JS would like to thank Dr. Mark Bundy of Baltimore, MD for his hospitality during the author's two week visit measuring position coordinates at the Space Telescope Science Institute. One of us (GK) wishes to acknowledge that this research was partially supported by a grant from NASA administered by the AAS.

JAS, VHCh, LKE and VTA wish to thank V. Vlasyuk, for use of his data conversion software and H. Tovmassian for helpful discussion. We thank N. Serafimovich for her help in preparing the paper. JAS, LKE and VTA were partially supported by the research grant No. 97-02-17168 from the Russian Foundation for Basic Research and No. 1.2.2.2 grant from Russian State program "Astronomy". VHCh was supported by the CONACYT research grant No. 28499-E.

We thank the referee Dr. M.P. Véron for a number a valuable comments which helped to improve the paper. 


\section{References}

Bowen D.V., Osmer S.J., Blades J.C., Tytler D., Cottrell L., Fan X.-M., Lanzetta K.M., 1994, AJ 107, 461

Chavushyan V.H., Stepanian J.A., Balayan S.K., Vlasyuk V.V., 1995, Astron. Lett. 21, 894

Green R., Morrill M.E., 1978, PASP 90, 601

Green R., Schmidt M., Liebert J., 1986, ApJS 61, 305

Heckman O., Dickevoss W., Kox H., Grunter A., Brosterhus E., 1975, AGK 3 star catalog of Positions and Proper Motions North of -2.5 degree. Hamburg-Bergedorf, Hamburg

Kirhakos S., Sargent W.L.W., Schneider D.P., et al., 1994, PASP 106, 646

Lieske J.H., 1979, A\&A 73, 282

Markarian B.E., 1967, Astrofizica 3, 55

Markarian B.E., Lipovetsky V., Stepanian J.A., 1981, Astrofizica 17, 619

Markarian B.E., Stepanian J.A., 1983, Astrofizica 19, 639

Markarian B.E., Stepanian J.A., 1984a, Astrofizica 20, 21
Markarian B.E., Stepanian J.A., 1984b, Astrofizica 20, 513

Markarian B.E., Stepanian J.A., Erastova L.K., 1985, Astrofizica 23, 439

Markarian B.E., Stepanian J.A., Erastova L.K., 1986, Astrofizica 25, 345

Nelson L.R., Kojoian G., Seal J., Pearson D.H., 1988, AJ 95, 1678

Stepanian J.A., Lipovetsky V.A., Erastova L.K., 1988, Astrofizica 29, 247

Stepanian J.A., Lipovetsky V.A., Erastova L.K., 1990, Astrofizica 32, 441

Stepanian J.A., 1994, D. Sci. Thesis, Nizhnij Arkhiz

Sanduleak N., Pesch P., 1987, ApJS 63, 809

Smith M.G., 1975, ApJ 202, 591

Schneider D.P., Bahcall J.N., Saxe D.H., et al., 1992, PASP 104, 678

Seal J., Chavez D., Kojoian G., 1990, AJ 100, 1028

Simpson C.E., 1990, PASP 102, 478

Véron-Cetty M.-P., Véron P., 1996, A\&AS 115, 97 